\title{
Plasma Coagulation Controller: A Low- Power Atmospheric Plasma Source for Accelerated Blood Coagulation
}

\author{
G. De Masi, ${ }^{a, b, *}$ C. Gareri, ${ }^{b}$ L. Cordaro, ${ }^{a}$ A. Fassina, ${ }^{a}$ P. Brun,,${ }^{c}$ B. Zaniol, ${ }^{a}$ \\ R. Cavazzana, ${ }^{a}$ E. Martines, ${ }^{a}$ M. Zuin, ${ }^{a}$ G. Marinaro, ${ }^{b}$ S. De Rosa, ${ }^{b}$ \& C. Indolfi ${ }^{b}$ \\ aConsorzio RFX, Padova, Italy; 'Dipartimento di Scienze Mediche e Chirurgiche, Università \\ Magna Graecia di Catanzaro, Italy; 'Dipartimento di Medicina Molecolare, Unità di Microbiologia, \\ Università di Padova, Italy \\ *Address all correspondence to: G. De Masi, Consorzio RFX, C.so Stati Uniti, 4 - 35127, Padova, Italy; \\ Tel.: +390498295009, E-mail: gianluca.demasi@igi.cnr.it
}

\begin{abstract}
In this contribution we present the main features of the plasma coagulation controller (PCC) device, a cold atmospheric pressure plasma source based on the dielectric barrier discharge (DBD) scheme, specifically designed for accelerating blood coagulation. The device is controlled by a microcontroller and can be used to explore different operational parameters in terms of discharge repetition rate $(1-20 \mathrm{kHz})$ and applied voltage $(2-8 \mathrm{kV})$. Helium is used as a working gas. Effective current measured on a metallic target is of the order of $1 \mathrm{~mA}$ and, thus, is suitable for application on the human body. Emission spectroscopy analysis is performed: the presence in the plasma of reactive oxygen species (ROS) and reactive nitrogen species (RNS) is highlighted in the different operational regimes. We present the results of multiple tests using the device in different biological processes, including reactive species production, bacterial removal, and in vitro blood coagulation. Interestingly, tests on blood samples exposed to the helium plasma at different times show a significant reduction of the coagulation compared to control samples.
\end{abstract}

KEY WORDS: plasma medicine, atmospheric pressure plasma applications, dielectric barrier discharge, biomedical applications

\section{INTRODUCTION}

Plasma medicine is an emerging field among the broad spectrum of plasma science applications. It deals with the use of cold plasmas produced at atmospheric pressure to obtain a therapeutic effect on living matter. The most studied fields of application at present include disinfection, ${ }^{1}$ wound healing, ${ }^{2}$ blood coagulation, ${ }^{3-5}$ and cancer cell treatment. ${ }^{6,7}$ The driving idea behind plasma medicine is to perform a very localized and nonthermal (few watts of power) treatment of the human/animal body substrate. To achieve this goal, different plasma source schemes have been developed, the most diffused being the radio frequency ${ }^{1}$ and the dielectric barrier discharge (DBD) concepts (described in section 2). In both cases, a neutral gas is ionized by applying a time-variable electric field between two electrodes. This can be used to produce a plasma that does not reach the thermodynamic equilibrium being characterized by highly energetic electron populations and "cold" ions (and neutral particles) close to room temperature. At atmospheric pressure the main gas (typically helium or argon) mixes with surround- 
ing air, so the produced plasma is enriched with ROS and RNS as a result of the chemical reactions induced by the energetic electrons. Though the mechanisms behind the plasma-living matter interaction are not fully understood, the aforementioned excited molecules, together with the UV radiation, the electromagnetic field, and the electric charged particles (ion and electrons) are considered as the main agents of the observed therapeutic effect.

In this contribution, we present a novel plasma medicine tool, based on a DBD scheme, specifically designed for accelerating blood coagulation.

\section{PLASMA COAGULATION CONTROUER}

A non-thermal plasma source at atmospheric pressure has been realized upon exploiting the concept of DBD: plasma is generated by applying a pulsed electric field at high voltage between two electrodes separated by a dielectric material (Fig. 1) with the goal of limiting the current associated to the breakdown. On the plasma coagulation controller (PCC) device, high voltage $\left(\mathrm{V}_{\text {app }}\right.$ range is $\left.2-8 \mathrm{kV}\right)$ is achieved through a ferrite core coil mounted on the source head and directly connected to the central electrode covered by a Pyrex tube (dielectric). A metallic ring housed on an external concentric Pyrex tube acts as a grounded electrode. The device is controlled by an Arduino Leonardo microcontroller that makes the system extremely flexible: applied voltage and repetition rate ( $\mathrm{f}$ range is $1-20 \mathrm{kHz}$ ) can be easily modified through a graphic interface. Either helium or argon can be used as a working gas for the PCC. However, in this contribution we will present only helium plasma treatment.

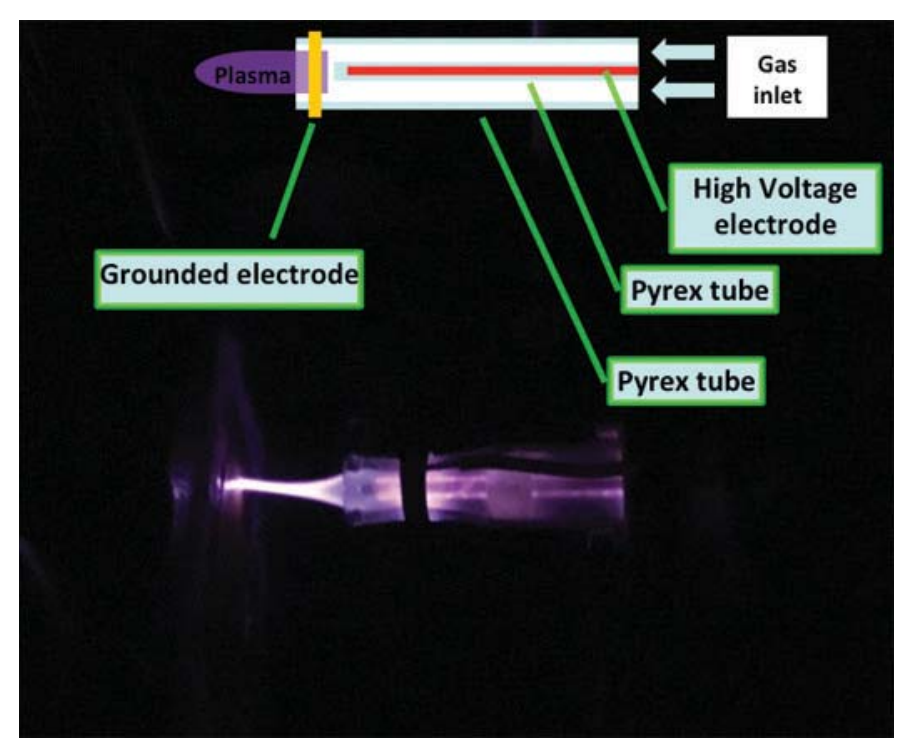

FIG. 1: Schematic of the DBD concept (top) and real picture of the helium plasma plume produced by the PCC device (bottom) 
The device has been characterized using a fast multichannel oscilloscope, a passive high voltage probe and an AC current probe. In Fig. 2, the time trace of the applied voltage on the central electrode is shown. Current $I_{\text {plate }}$ has been also measured on a metallic grounded plate placed at a distance of $1 \mathrm{~cm}$ from the source head. The current waveform is also shown in Fig. 2. Effective current on the plate (averaged over $1 \mathrm{~ms}$ of application) can be estimated on the order of:

$$
\frac{1}{\Delta \mathrm{t}} \int_{\Delta t=1 m s} I_{\text {plate }} d t=1 \mathrm{~mA}
$$
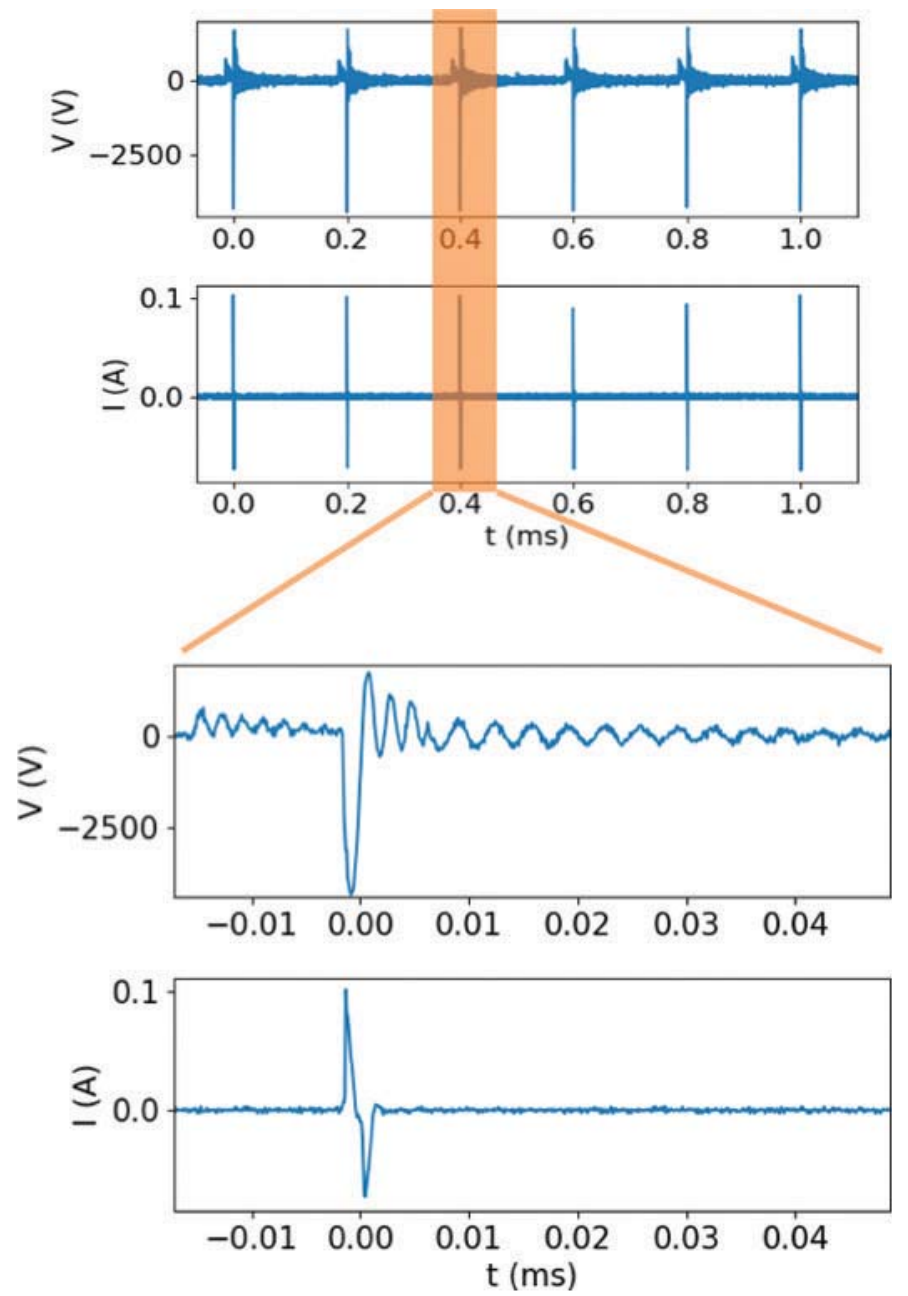

FIG. 2: Time trace of the applied voltage $V_{\text {app }}$ on the central electrode and of the current Iplate measured on the grounded metallic plate placed in front of the source head (top). Zoom on a single pulse is also shown (bottom)

Volume 8, Issue 3, 2018 
The effective power on the plate is on the order of a few watts (depending on the operational setup).

The high flexibility of PCC allows investigation of different operational scenarios by varying applied voltage or repetition rate. According to the measured voltage, we defined three operational conditions (called high, standard, and low) to study chemical composition and effect on bacteria and blood samples. The three scenarios feature the following parameters:

- High: $\mathrm{V}_{\mathrm{app}}=8 \mathrm{kV} ; \mathrm{f}=2 \mathrm{kHz}$

- Standard: $\mathrm{V}_{\text {app }}=6 \mathrm{kV} ; \mathrm{f}=5 \mathrm{kHz}$

- Low: $\mathrm{V}_{\text {app }}=4 \mathrm{kV} ; \mathrm{f}=10 \mathrm{kHz}$

\section{SPECTROSCOPIC ANALYSIS}

Plasma emission was analyzed using a PI IsoPlane spectrometer of $320 \mathrm{~mm}$ focal length, equipped with a 300 and a $2400 \mathrm{~g} / \mathrm{mm}$ gratings, and coupled to a PIXIS camera with $2048 \times 512$ square pixels of $13 \mu \mathrm{m}$. The system resolution depends on the grating. The first one is $1.5 \mathrm{~nm}$, and the second one is $0.087 \mathrm{~nm}$ (at $300 \mathrm{~nm}$ ) when using an entrance slit of $50 \mu \mathrm{m}$. The light was collected by an optical head consisting of a fused silica lens with a focal length of $10 \mathrm{~mm}$ and a diameter of $8 \mathrm{~mm}$ diameter, focusing the plasma image on a $400 \mu \mathrm{m}$ core optical fiber. The fiber, also made of fused silica, was $7 \mathrm{~m}$ long, and the other end was coupled to the spectrometer entrance slit. The spectral response of the entire diagnostic had been measured from $350 \mathrm{~nm}$ to $750 \mathrm{~nm}$, using an integrating sphere. The optical head was placed perpendicular to the plasma plume just outside the plasma source head.

The entire spectrum between $330 \mathrm{~nm}$ and $800 \mathrm{~nm}$ has been acquired with the low resolution grating (Fig. 3). Integrating time is on the order of few seconds. Clearly visible are the $\mathrm{N}_{2}$ ro-vibrational lines of the second positive system (SPS) and the $\mathrm{N}_{2}^{+}$lines of the first negative system (FNS), the $\mathrm{OH}$ band around $300 \mathrm{~nm}$, and the most intense lines of helium I and the oxygen I triplet at $777 \mathrm{~nm} . \mathrm{H}_{\alpha}$ and $\mathrm{H}_{\beta}$ lines are also above the noise level.

The rotational temperatures of the $\mathrm{OH}$ and $\mathrm{N}_{2}$ molecules were deduced from their rotational spectra. ${ }^{8}$ The two bands of $\mathrm{OH}$ at around $309 \mathrm{~nm}$ and and the $\mathrm{N}_{2}$ band at $337.1 \mathrm{~nm}$ have been simulated for different rotation temperatures, accounting for the diagnostic instrumental function and the dispersion. The temperature corresponding to the best match between the experimental spectrum and the simulations was found by minimizing the integral of their squared differences. An example of the measured and simulated best-fitting spectra is shown in Fig. 4 for the $\mathrm{OH}$ band.

The rotational temperatures of the two molecules were fully compatible, and ranged between $317 \pm 22 \mathrm{~K}$ and $279 \pm 27 \mathrm{~K}$.

\section{BIOLOGICALTESTS}

The antibacterial effect of the plasma produced with PCC has been tested by treating gram-negative and gram-positive bacterial strains, usually involved in wound infections: 


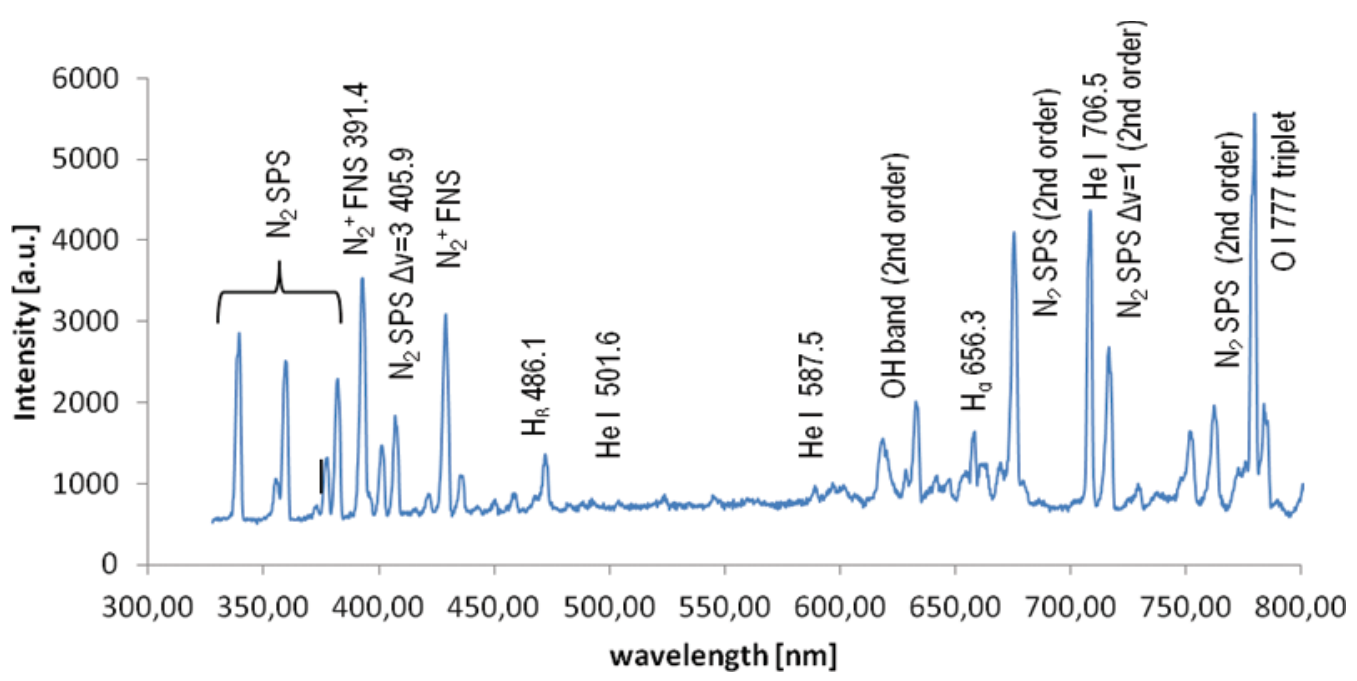

FIG. 3: Emission spectrum in the range (330-800 nm) of the helium plasma plume, measured $1 \mathrm{~mm}$ from the source head

Escherichia coli, Staphylococcus aureus, and Pseudomonas aeruginosa. Bacteria were grown in the proper medium at $37^{\circ} \mathrm{C}$. On the day of the experiment, $500 \mu \mathrm{L}$ of the culture were transferred to $5 \mathrm{~mL}$ of fresh medium and incubated under continuous shaking until the absorbance at $590 \mathrm{~nm}$ was 0.1 . Bacteria were then serially diluted to obtain suspension concentrations of 106 colony-forming units (CFU) per mL. Afterwards, $200 \mu \mathrm{L}$ of the solutions containing various amounts of microbes were placed in 24-well tissue culture plates (NUNC, Roskilde, Denmark; growth area $2 \mathrm{~cm}^{2}$, and well volume $3.5 \mathrm{~mL}$ ).

Then bacterial cultures were exposed to helium plasma produced with PCC for different time periods. Bacterial vitality was evaluated by counting colonies. As shown in Fig. 5, relative vitality of bacteria dropped to less than $50 \%$ after only $15 \mathrm{~s}$ of plasma

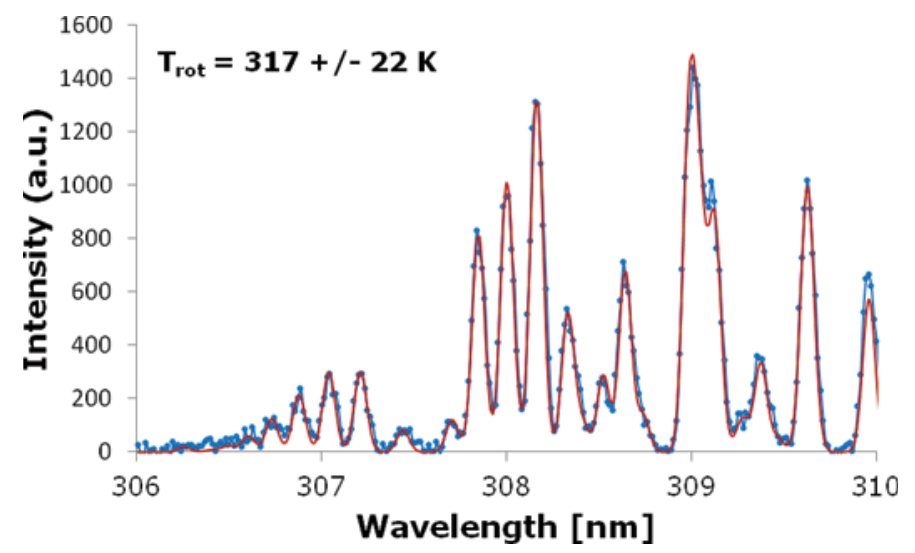

FIG. 4: Measured (blue dots) and simulated best fitting (red line) spectra of the $\mathrm{OH}$ band

Volume 8, Issue 3, 2018 
exposure at the standard condition and kept decreasing over time until reaching almost $100 \%$ after 2 min (see Fig. 5).

We also compared the bacteria response to the different PCC operational scenarios. Figure 6 shows the result on different bacteria after $15 \mathrm{~s}$ of PCC treatment. No significant differences have been observed among the different operational setups. As a control, a different plasma source (a RF source ${ }^{1,9}$ ) was used and reported in the same plot. Interestingly, PCC shows a faster bactericidal effect than RF on all the treated bacterial colonies.

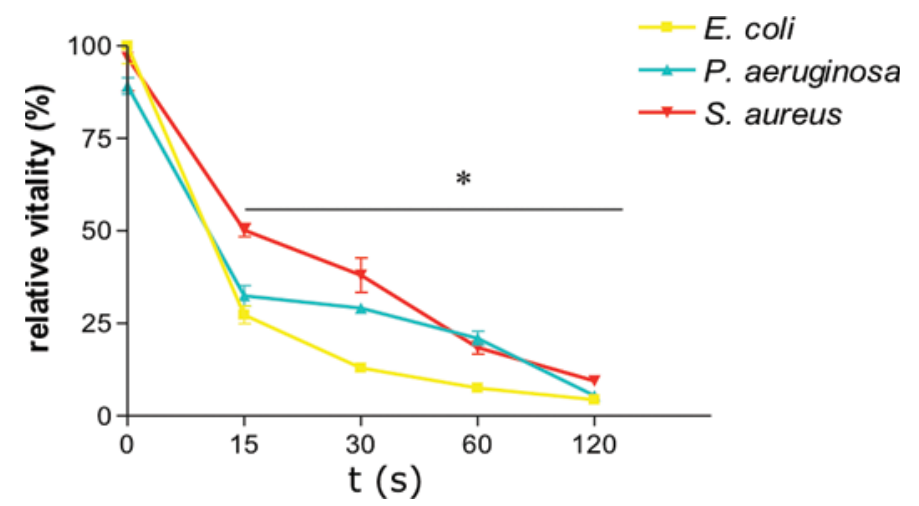

FIG. 5: Relative vitality of three different bacterial colonies treated under PCC (standard condition) irradiation for different time points

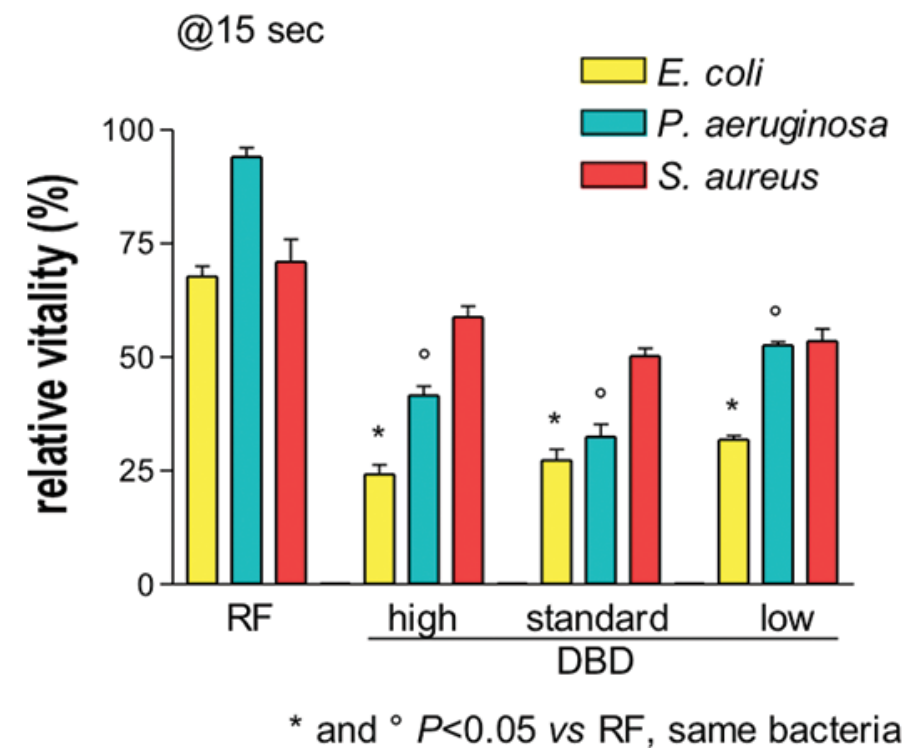

FIG. 6: Relative vitality of different bacteria colonies after plasma treatment of $15 \mathrm{~s}$ (left). Results with RF plasma treatment are also shown 
Using fluorescence-activated cell sorting (FACS) analysis we also studied the intracellular production of ROS and nitric oxide (NO) molecules as a response to the PCC exposure in human fibroblasts. Figure 7 shows the percentage of cells positive for the presence of ROS (left) and NO (right) for the control (cells exposed to air), with treatment at standard conditions for different time points: $15 \mathrm{~s}, 30 \mathrm{~s}, 1 \mathrm{~min}$, and $2 \mathrm{~min}$. The presence of intracellular ROS increased according to the treatment duration, suggesting a possible link with PCC action. On the other side, NO behavior displayed a sudden increase after only 15 $\mathrm{s}$ of treatment, but slightly decreased for longer treatments. The detailed cellular mechanisms involving in ROS and NO production will be further investigated in the future.

\section{ACCELERATED BLOOD COAGULATION: IN VIRO STUDIES}

Finally, the ability of PCC to accelerate blood coagulation in vitro has been tested repeatedly.

Blood samples were withdrawn from patients following anticoagulant therapy. In this setting, recent clinical studies have underlined the importance of maintaining an optimal anticoagulation state also during surgery to warrant patient protection. In fact, the incidence of thromboembolic events is higher around the time of the procedure. ${ }^{10}$ Nonetheless, maintenance of periprocedural anticoagulation raises the bleeding risk. In this setting, the availability of a device that could facilitate hemostasis and prevent infections around the peri-procedural phase would be very helpful. Blood samples were withdrawn and stored in Vacutainer tubes containing ethylenediaminetetraacetic acid (EDTA) and kept at $4{ }^{\circ} \mathrm{C}$ until they were processed (2-4 h). $200 \mu \mathrm{L}$ of blood were transferred to a single well of a 24-well plate, and $20 \mu \mathrm{L}$ of $4 \mathrm{M} \mathrm{CaCl}_{2}$ was added to contrast the EDTA effect. Each well was immediately stimulated with its specific treatment for $15 \mathrm{~s}, 30 \mathrm{~s}$, or $1 \mathrm{~min}$, respectively. Control (CTRL) samples were exposed to air at room temperature (no treatment). After the treatment, the blood was gently removed, and the
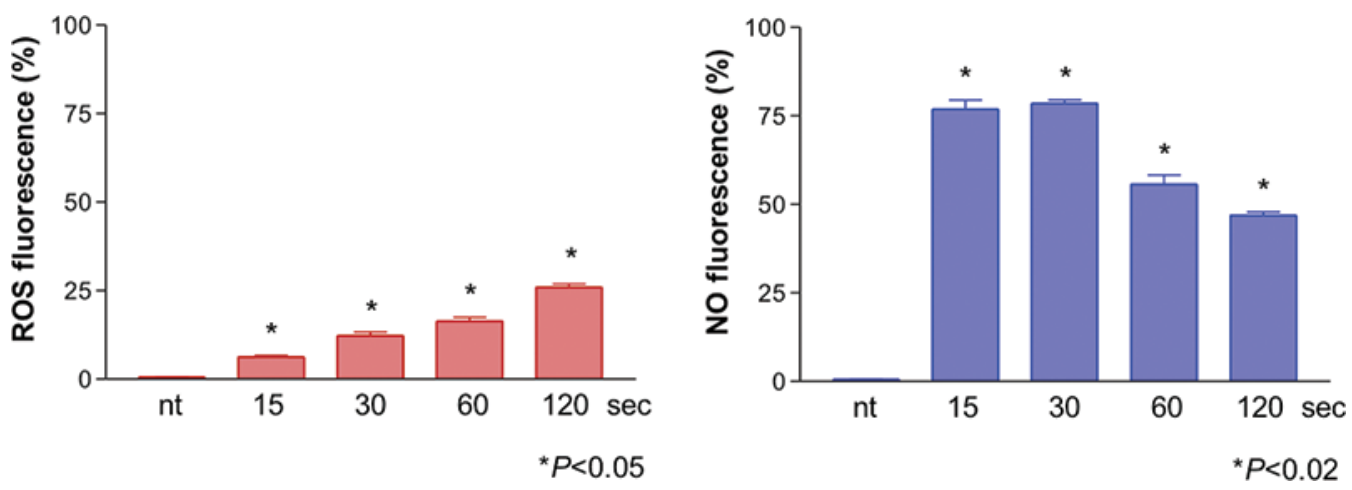

FIG. 7: Percentage of human fibroblasts cells that are positive for the presence of ROS and NO molecules after PCC exposure (standard conditions) for different time points. Left bars in each plot refer to control cells exposed to air (nt)

Volume 8, Issue 3, 2018 


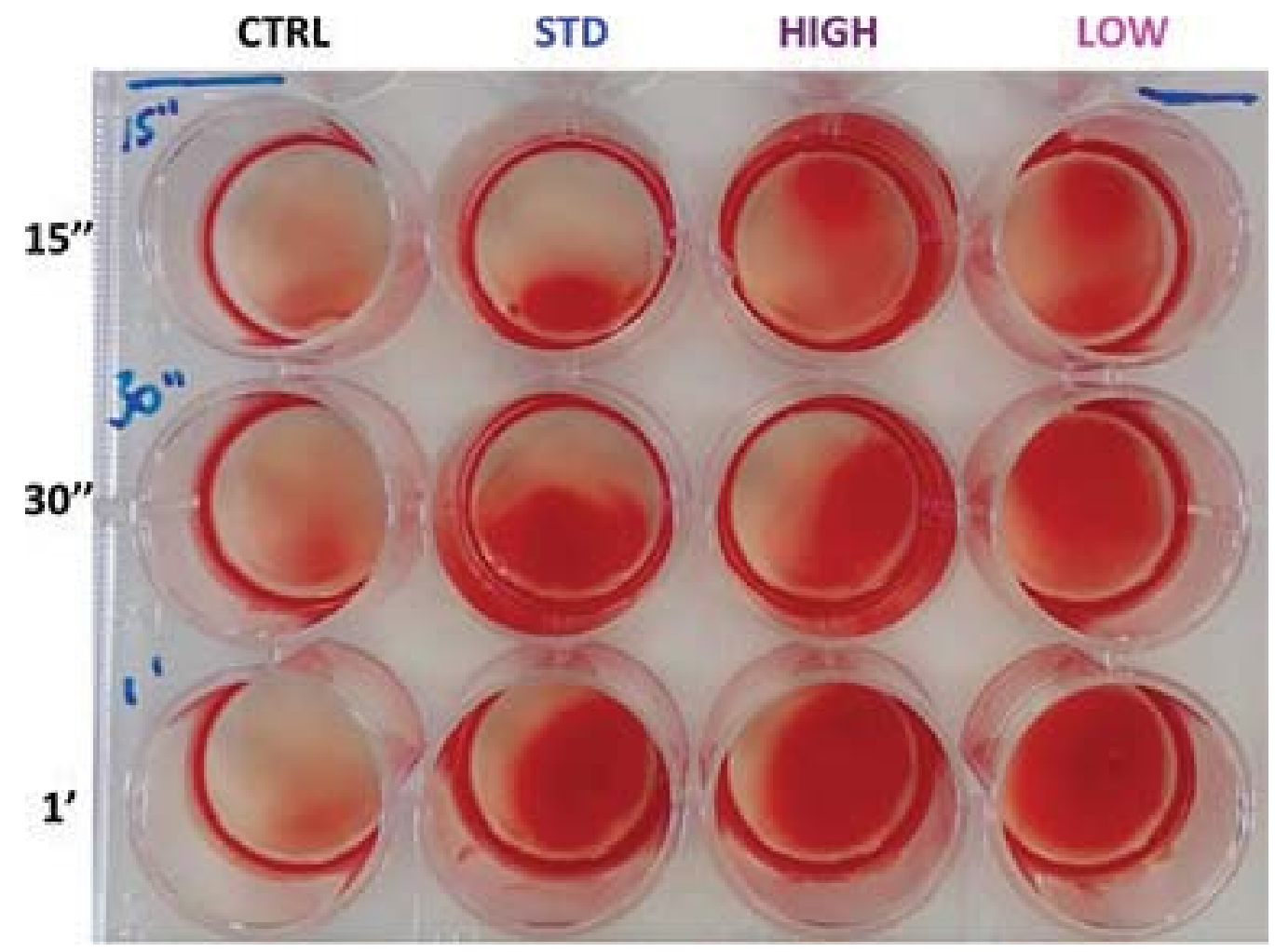

FIG. 8: Blood clots formed in the well surface after PCC treatment for different time points and different operational setup: control (CTRL), Standard (STD), High, and Low

presence of blood clots on the well surface evaluated immediately (data not shown) and at $24 \mathrm{~h}$ after the treatment. Figure 8 shows a representative image of a plate $24 \mathrm{~h}$ posttreatment. Each blood clot was photographed and the image measured using a customized MatLab (MathWorks, Inc.) script. The results were analyzed using GraphPad (GraphPad Software). As shown in Fig. 9, all the treatments showed a large increase in blood coagulation (expressed as area of coagulated blood remaining in each well) ( $\mathrm{N}=$ 10). Significant differences between the samples and the controls were found by using one-way analysis of variance (ANOVA) (15 s: $p<0.0099 ; 30 \mathrm{~s}: p<0.0007$; 1 min: $p<$ 0.0025). The PCC treatment significantly enhanced blood coagulation. No significant differences were observed among the three treatments. The results are displayed as mean of the replicates \pm standard error mean.

\section{CONCLUSION}

We present a newly developed plasma medicine tool, specifically designed to induce and enhance blood coagulation. A first prototype, whose main features are presented in 


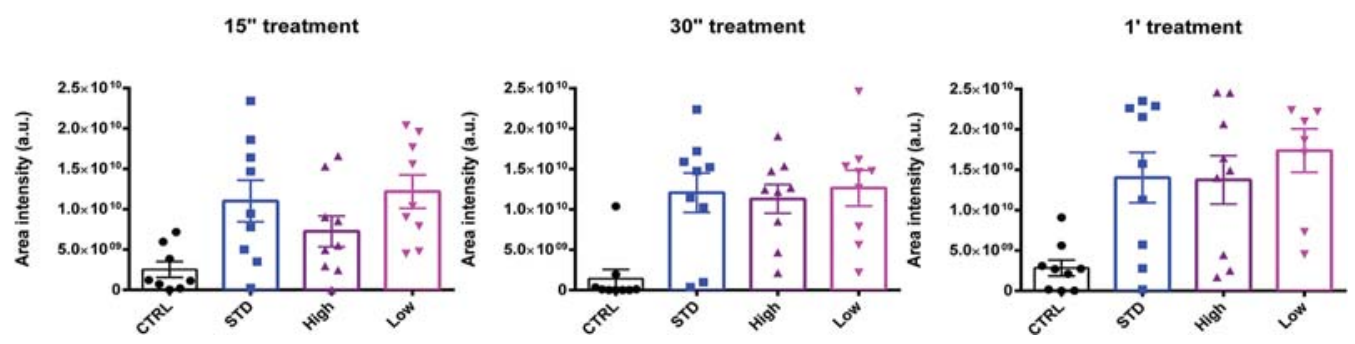

FIG. 9: Coagulation area intensity $24 \mathrm{~h}$ after PCC treatment. A one-way ANOVA test has been used. All treatments results were significantly different from the controls. $15 \mathrm{~s}: p<0.0099 ; 30 \mathrm{~s}$ : $p<0.0007$; 1 min: $p<0.0025$

this work, has been built, characterized with spectroscopic analysis, and successfully tested with different biomedical procedures. The presence of different chemical species that are likely to be involved in the interaction with living matter has been highlighted. Tests on different bacterial colonies demonstrated a clear antibacterial effect of the PCC treatment. Moreover, in vitro studies on blood samples showed a significant acceleration of blood coagulation in PCC-treated samples compared to controls. Further in vitro tests will be performed to confirm these results and to investigate the detailed molecular mechanisms underlying the effect exerted by PCC on blood coagulation.

\section{ACKNOWHDGMENTS}

This work has been conducted as a part of the three-year research project named "A novel plasma medicine tool for accelerated heamostasis," which has received funding from the "Fondazione con il Sud" within the call "Brains2South." The authors thank Dr. H. Sakakita and Dr. Y. Ikehara for their valuable collaboration and their constant support.

\section{REFERENCES}

1. Brun P, Brun P, Vono M, Venier P, Tarricone E, Deligianni V, Martines E, Zuin M, Spagnolo S, Cavazzana R, Cardin R, Castagliuolo I, La Gloria Valerio A, Leonardi A. Disinfection of ocular cells and tissues by atmospheric-pressure cold plasma. PLoS One. 2012;7(3):e33245.

2. Haertel B, von Woedtke T, Weltmann KD, Lindequist U. Non-thermal atmospheric-pressure plasma possible application in wound healing. Biomol Ther (Seoul). 2014;22:477-90.

3. Sakakita H, Ikehara Y. Irradiation experiments on a mouse using mild-plasma generator for medical applications. Plasma Fusion Res. 2010;5:S2117.

4. Ikehara Y, Sakakita H, Shimizu N, Ikehara S, Nakanishi H. Formation of membrane-like structures in clotted blood by mild plasma treatment during hemostasis. J Photopolym Sci Technol. 2006;26:425.

5. Fridman G, Peddinghaus M, Balasubramanian M, Ayan H, Fridman A, Gutsol A, Brooks A. Blood coagulation and living tissue sterilization by floating-electrode dielectric barrier discharge in air. Plasma Chem Plasma Process. 2013;26(4):555-72.

Volume 8, Issue 3, 2018 
6. Graves DB. Reactive species from cold atmospheric plasma: implications for cancer therapy. Plasma Process Polym. 2014;11:1120-7.

7. Martines E, Brun P, Artico R, Brun P, Cavazzana R, Cordaro L, De Masi G, Fischetto D, Zuin A, Zuin M. Role of intracellular RONS in plasma-based cancer treatment. Proceedings of the 23rd International Conference on Phenomena in Ionized Gases; 2017 July 9-14; Estoril, Portugal; p. 368.

8. de Izarra C. UV OH spectrum used as a molecular pyrometer. J Phys D: Appl Phys. 2000;33:16971704.

9. Martines E, Zuin M, Cavazzana R, Gazza E, Serianni G, Spagnolo S, Spolaore M, Leonardi A, Deligianni V, Brun P, Aragona M, Castagliuolo I, Brun P. A novel plasma source for sterilization of living tissues. New J Phys. 2009;11:115014.

10. Santarpia G, De Rosa S, Polimeni A, Giampà S, Micieli M, Curcio A, Indolfi C. Efficacy and safety of non-vitamin $\mathrm{K}$ antagonist oral anticoagulants versus vitamin $\mathrm{K}$ antagonist oral anticoagulants in patients undergoing radiofrequency catheter ablation of atrial fibrillation: a meta-analysis. PLoS One. 2015;10(5):e0126512. 